\title{
Patient-specific surgical options for breast cancer- related lymphedema: technical tips
}

\author{
Jin Geun Kwon, Dae Won Hong, Hyunsuk Peter Suh, Changsik John Pak, Joon Pio Hong \\ Department of Plastic and Reconstructive Surgery, Asan Medical Center, University of Ulsan College of Medicine, Seoul, Korea
}

In order to provide a physiological solution for patients with breast cancer-related lymphedema (BCRL), the surgeon must understand where and how the pathology of lymphedema occurred. Based on each patient's pathology, the treatment plan should be carefully decided and individualized. At the authors' institution, the treatment plan is made individually based on each patient's symptoms and relative factors. Most early-stage patients first undergo decongestive therapy and then, depending on the efficacy of the treatment, a surgical approach is suggested. If the patient is indicated for surgery, all the points of lymphatic flow obstruction are carefully examined. Thus a BCRL patient can be considered for lymphaticovenous anastomosis (LVA), a lymph node flap, scar resection, or a combination thereof. LVA targets ectatic superficial collecting lymphatics, which are located within the deep fat layer, and preoperative mapping using ultrasonography is critical. If there is contracture on the axilla, axillary scar removal is indicated to relieve the vein pressure and allow better drainage. Furthermore, removing the scars and reconstructing the fat layer will allow a better chance for the lymphatics to regenerate. After complete removal of scar tissue, a regional fat flap or a superficial circumflex iliac artery perforator flap with lymph node transfer is performed. By deciding the surgical planning for BCRL based on each patient's pathophysiology, optimal outcomes can be achieved. Depending on each patient's pathophysiology, LVA, scar removal, vascularized lymph node transfer with a sufficient adipocutaneous flap, and simultaneous breast reconstruction should be planned.

Keywords Lymphedema / Breast cancer lymphedema / Lymphaticovenous anastomosis / Lymph node transfer
Correspondence: Joon Pio Hong Department of Plastic and

Reconstructive Surgery, Asan Medical Center, University of Ulsan College of Medicine, 88 Olympic-ro 43-gil, Songpa-gu, Seoul 05505, Korea Tel: $+82-2-3010-3600$ Fax: +82-2-476-4741 E-mail: joonphong@amc.seoul.kr

Received: December 11, 2020 • Revised: February 16, 2021 • Accepted: April 9, 2021

pISSN: 2234-6163 • elSSN: 2234-6171 • https://doi.org/10.5999/aps.2020.02432・Arch Plast Surg 2021;48:246-253

\section{INTRODUCTION}

Breast cancer-related lymphedema (BCRL) occurs in one of every five patients $(21.4 \%)[1,2]$. The incidence of lymphedema during or after breast cancer treatment further debilitates patients and increases the challenge of achieving a symptom-free daily life [3]. In addition, the burden of knowing that the correction or treatment may not alleviate the symptoms related to lymphedema further frustrates patients [4].

To achieve physiological improvement of lymphedema, surgical reconstruction rather than decongestive therapy may be preferred. In order to provide a physiological solution, one must understand where and how the pathology of lymphedema occurred. Even though there is no conclusively established pathophysiology of BCRL, physical interruption of flow, radiation, obesity, and other factors have been proposed as major causes of 
BCRL [4]. Physiological treatments, such as lymphaticovenous anastomosis (LVA) and vascularized lymph node transfer, have been developed to address the factors of interruption and absence of the lymphatic system [5].

These reconstructive treatments address the pathology directly, which has instigated a shift of the treatment paradigm from symptom management to physiological treatment. This is an ongoing field of surgery, where further research is needed to determine both the efficacy and indications of these procedures. However, a consensus is slowly building based on the physical existence of lymphatic vessels. As an imaging technique, indocyanine green (ICG) enables the visualization of lymphatic vessels and plays a key role in determining the indications for lymphatic surgery. Unfortunately, the limited depth of ICG penetration means that it cannot be used to determine the applicability of various procedures for advanced-stage lymphedema. Although advanced-stage lymphedema may involve further dysfunction of the lymphatic system, there is not enough evidence to determine whether it can be surgically corrected. In our experience, the lymphatic vessels may still be functional even in the most advanced cases [6]. Therefore, the approach to each patient should be carefully planned and individualized.

At the authors' institution, the treatment plan is made individually based on the patient's symptoms and relative factors. Most early-stage patients first undergo decongestive therapy, and then, depending on the efficacy of the treatment, a surgical approach is suggested [4]. If a patient is indicated for surgery, a careful examination is performed to determine all the points of lymphatic flow obstruction. Thus, patients with BCRL, can be considered for LVA, a lymph node flap, scar resection, or a combination thereof. The aim of this paper is to introduce a treatment planning algorithm for BCRL patients based on each patient's pathophysiology.

\section{EVALUATION OF THE PATIENT'S PRESENTATION}

In lymphedema, interstitial fluid accumulates due to a lack of drainage of the lymphatic system. Ideally, the best approach would be to reconstruct the lymphatic system itself, but doing so can be difficult. Thus, in order to bypass the obstruction, a lymphaticovenous shunt is created distal to the obstruction, restoring the flow of the lymphatics. The evaluation should start by identifying the obstruction point to address the issues involved.

First, a careful history should be taken, with careful consideration of previous oncologic surgery, including the extent of axillary lymph node dissection, history of seroma, infection, hema- toma, and radiation therapy, as these events can cause severe scarring and fibrosis of the axilla, increasing the risk for lymphedema.

A physical evaluation of the severity of fibrosis of the axilla is then performed. Based on the findings of this evaluations, patients are categorized into three presentations: group 1, patients whose axillary continuity is well maintained with sufficient soft tissue; group 2, patients showing axillary continuity of the soft tissue but with firm and scarred tissue; and group 3, patients whose axillary continuity is completely disconnected and who show muscle to skin tightness. Evaluating the limitation of the axillary range of motion can provide helpful information. These findings determine the course of the axilla.

Finally, the patient undergoes multiple diagnostic modalities, such as lymphoscintigraphy, magnetic resonance lymphangiography, ultrasonography, and ICG imaging, depending on their physical status $[7,8]$. Specific descriptions of these modalities will not be presented in this paper.

\section{TREATMENT AND ALGORITHM}

\section{Lymphaticovenous anastomosis}

The basic tool for upper limb lymphedema treatment is LVA [3]. The role of LVA for BCRL patients is superb in comparison to its role for lower limb lymphedema patients. As gravity is less effective in the upper limb than in the lower limb, the function of the lymphatics is relatively well preserved. Therefore, even in advanced stages, there is a high likelihood of finding functioning lymphatics using ultrasonography [4].

LVA targets the superficial collecting lymphatics, which are located within the deep fat layer. Superficial lymphatics located within the dermis or superficial fat layer are precollectors that merge into the superficial collecting lymphatics. Therefore, by performing LVA to the superficial collecting lymphatics, the effect of LVA is maximized [9]. Among the numerous superficial collecting lymphatics, the ectatic lymphatics, which are transparent and overinflated with high-pressure lymphatic fluid, are the primary target selected for LVA. Since the ectatic lymphatics actively drain the lymphatic fluid, lymphatic fluid will drain through the LVA bypass pathway. In contrast, sclerotic lymphatics with hypertrophic, white walls have most likely lost their function of conveying lymphatic fluid [10]. Therefore, finding the ectatic lymphatics is the key to successful LVA.

To find the ectatic superficial collecting lymphatics, preoperative mapping using ultrasonography is critical. The superficial collecting lymphatics are usually located just below the superficial fascia. The key technique to view the target vessel is to apply mild compression and to view the patent lumen; while under 
high pressure, the lumen collapses, indicating that it is a lymphatic vessel [7]. This distinction is the key to separate lymphatics from veins, which collapse under weak pressure, and nerves, which have no lumen that can collapse. Since several lymphatics may be present, the surgeon should select the best-functioning lymphatics among those that are available. Functioning lymphatics that have ectasia show inflated circular lumina on ultrasonography. In addition, as sclerotic changes of the lymphatic wall are seen as hyperechoic walls on ultrasonography, it is recommended to choose lymphatics with thin and isoechoic walls.

Every superficial vein can be a candidate for LVA, but a vein with a valve proximal to the LVA is preferred as it will minimize backflow. Considering that LVA works based on the pressure gradient formed between the lymphatics and the vein, a vein with a proximal valve can maximize the pressure gradient [4]. In addition, if there is a vein with a branch, the branch is preferred for anastomosis due to the Venturi effect, which allows an in-
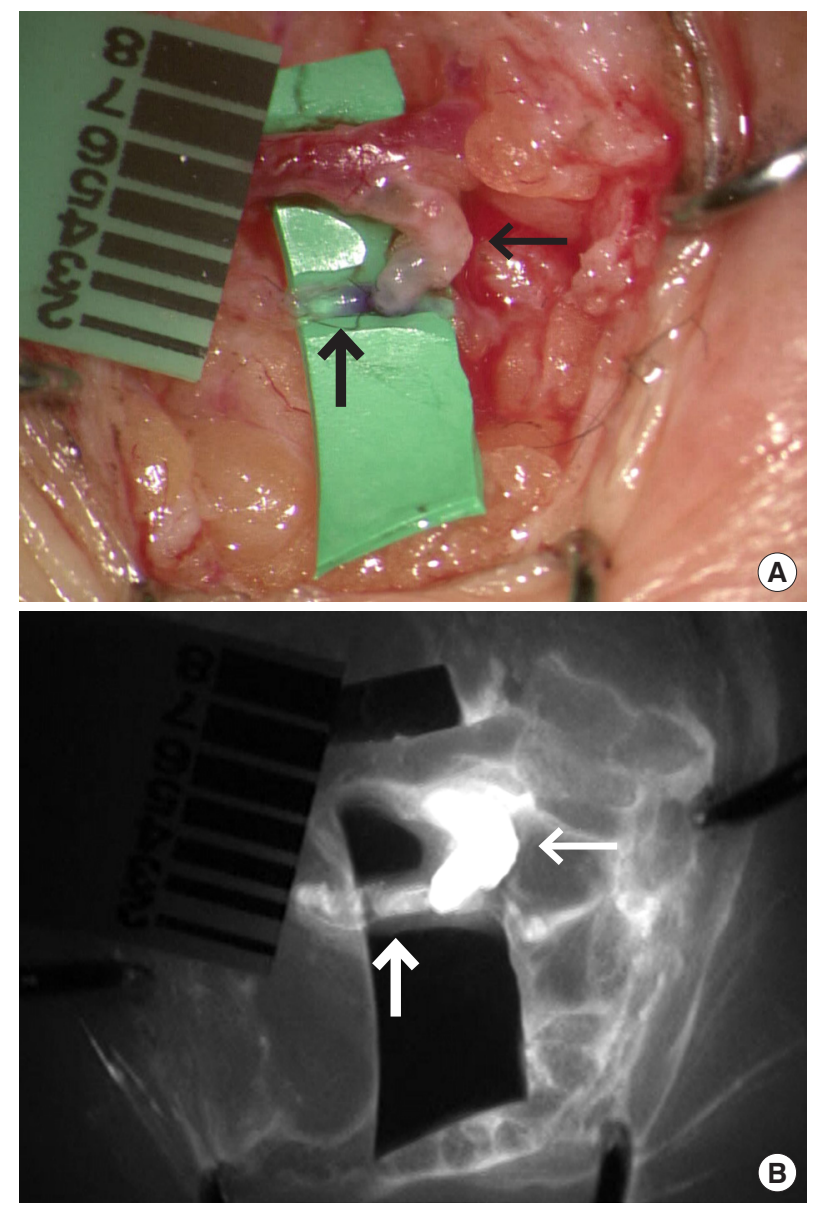

Fig. 1. After side-to-end lymphaticovenous anastomosis. A 0.4-mm size ectatic lymphatic was anastomosed to the vein in a side-to-end fashion. Thick arrow is lymphatics and thin arrow is superficial vein. (A) The color of the vein changed to transparent due to flow from the lymphatics. (B) Indocyanine green lymphangiography showing drainage of lymphatic fluid into the vein. crease in flow toward the main vein.

The methods of anastomosis vary from case to case. Among the end-to-end, end-to-side, side-to-end, and side-to-side anastomosis techniques, anastomosis of the side of the lymphatics to the end of the vein, and side-to-side anastomosis are preferred as ways to preserve the original lymphatic flow (Fig. 1) [11]. When there is a very large size discrepancy between the lymphatic and vein, side-to-side anastomosis can be easier than other anastomosis techniques (Fig. 2).

To optimize outcomes, meticulous anastomosis is required, without leakage or mistakenly sutures of the back wall [12]. The biggest obstacle during anastomosis is identifying the lymphatic wall and the patent lumen. Although some reports have advocated that more anastomoses can be better, the authors believe that one good side-to-end anastomosis can be as good as multiple end-to-end anastomoses. The reason for this is that the entire lymphatic system is connected to one continuous system,
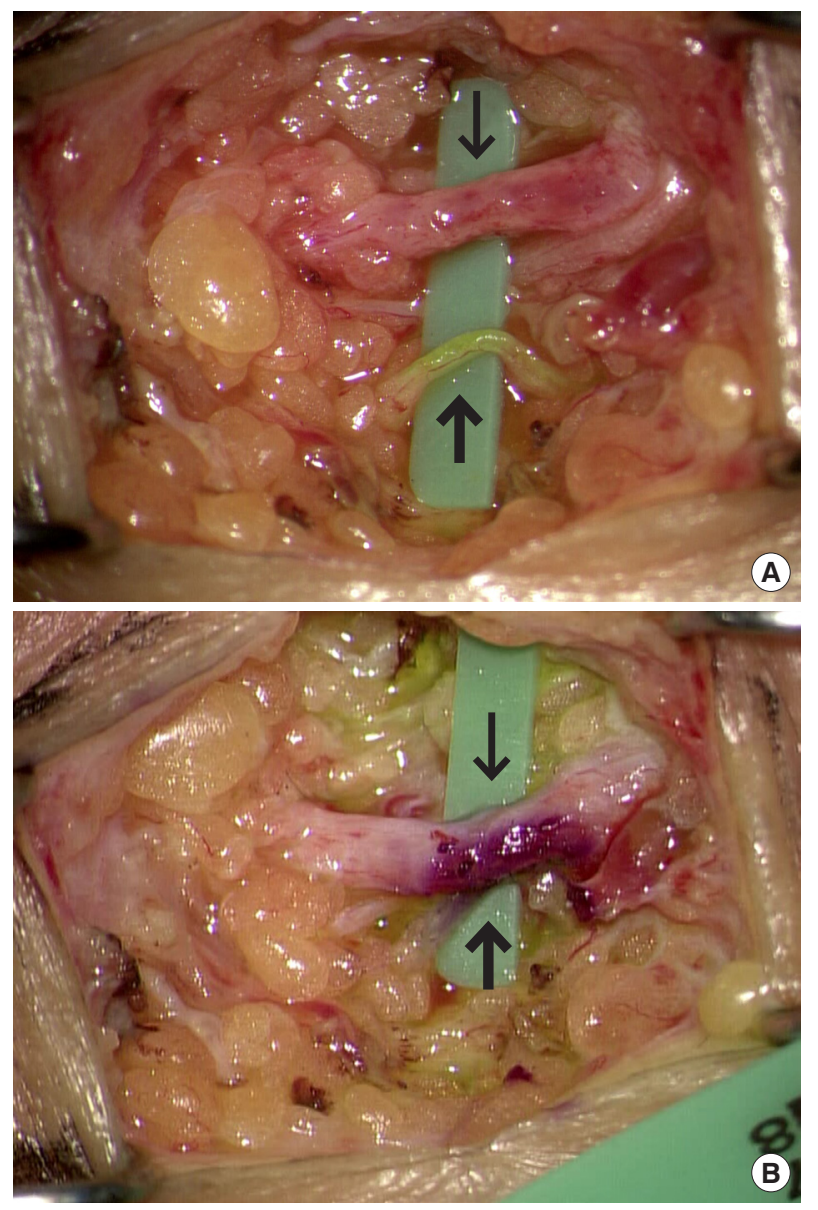

Fig. 2. Side-to-side lymphaticovenous anastomosis. (A) The vein of the upper limb had a larger caliber than that of the lower limb. The size discrepancy was problematic. (B) Side-to-side lymphaticovenous anastomosis can solve the size discrepancy, maintaining the physiological pathway of lymphatics. Thick arrow is lymphatics and thin arrow is superficial vein. 
and the valvular function of the lymphatics is impaired in lymphedema patients $[13,14]$.

The authors apply immediate compression on the surgical table. The target pressure is $25-35 \mathrm{mmHg}$, creating a higher-pressure gradient between the lymphatics and vein after LVA [15].

\section{Axillary scar removal and fat advancement flap}

Recent studies have shown that chronic fibrosis plays a key role in the pathophysiology of lymphedema [16,17]. When opening the axilla, one of the goals is to remove the scars/fibrosis completely or as much as possible [3]. We believe that scar removal relieves the vein pressure and allows better drainage. Therefore, removing the perivascular scarring surrounding the axillary vein is key. Furthermore, removing the scars and reconstructing the fat layer will make it more likely that the lymphatics will regenerate. However, removing scars from the axilla is not easy due to the complicated neurovascular anatomy of the axilla. The axillary vein is a landmark, as it is large and located more superficially than the axillary artery or brachial plexus. By dissecting the space below the pectoralis major muscle, the surgeon can easily reach the axillary vein. Once the axillary vein is seen, careful dissection is required to minimize the damage to the brachial nerve and axillary artery. The key to dissecting perivascular scarring is finding the layer between the scar tissue and the normal anatomy. Once this layer is found, dissection can be performed easily with minimal risk. In addition, special care is required not to injure the thoracodorsal system and lateral thoracic system, as they can be used for recipient vessels during vascularized lymph node transfer.

After the complete removal of scar tissue, the size of the axillary defect is evaluated. If the gap or defect is small enough (usually, when the long axis of the defect is less than $3 \mathrm{~cm}$ ), then regional transposition or advancement of the fat based on a random-pattern pedicle can be performed to obliterate the defect after scar removal. In our experience, most patients who could undergo regional fat flaps were not advanced cases. In patients who underwent breast-conserving surgery with sentinel lymph node dissection and had enough axillary soft tissue, the defect was easily covered with a regional fat flap. However, in most advanced cases, there was a large defect, for which a free flap was needed to obliterate and reconstitute the fat layer. In particular, patients who underwent extensive axillary lymph node dissection commonly required an additional free flap, as the boundary of scarring was extensive.

\section{Axillary scar removal and vascularized lymph node transfer}

After axillary scar removal, if there is a large defect, then vascu- larized lymph node transfer with abundant adipose tissue is required to reconstitute the continuity of the fat and soft tissue under the skin. Since the best reconstructive option is replacing like with like, reconstruction with flaps that have abundant fat and lymphatic structure is ideal [18].

During scar tissue removal, potential recipient vessels can be identified. The thoracodorsal artery and vein beneath the latissimus dorsi muscle, the serratus branch of the subscapular artery and vein, and the lateral thoracic vessels on the chest wall are commonly used in our practice. One should try to acquire a lengthy recipient vessel to facilitate easy anastomosis.

Although various donor sites can be used, the superficial circumflex iliac artery perforator flap is our workhorse flap for upper arm lymphedema due to its ease of dissection, the abundance of fat and lymph nodes, reliable pedicle anatomy, and minimal donor-related morbidity [4]. The location of lymph nodes and pedicles can be preoperatively evaluated using computed tomography angiography, magnetic resonance imaging, and ultrasonography $[7,19]$. To prevent iatrogenic lymphedema of the donor site, the lymph node located medial to the femoral artery is preserved and the lymph node located lateral to the femoral artery is harvested [4]. Reverse mapping with an isotope can be performed to further minimize the risk of iatrogenic lymphedema [20]. Single photon emission computed tomography is another modality to protect functioning lymph nodes from being harvested, but imposes an additional cost [4]. It is important to completely fill the defect with the flap tissue, as any remnant dead space will cause fibrosis of the soft tissue, again obstructing the fat-to-fat contact. Depending on the dimensions of the defect, the flap dimensions are carefully designed (Fig. 3A).

There are two choices regarding the flap elevation plane. A super-thin (superficial fascia plane) flap can reduce the risk of iatrogenic lower limb lymphedema, as only the harvested lymph node is included in the flap, whereas the rest of the lymphatic structure is preserved $[21,22]$. However, if this flap is not sufficient to completely obliterate the dead space, then additional fat can be added by harvesting the flap on the deep fascia (suprafascial plane), but doing so may increase the risk of iatrogenic lower limb lymphedema.

A skin incision is made until the superficial fascia is reached, usually beginning from the lateral lower margin of the flap. After identifying the superficial branch of the superficial circumflex iliac artery and superficial vein penetrating the superficial fascia, the rest of the flap is elevated. The flap is elevated from the lateral toward the medial side, and as the dissection on this plane reaches near the pedicle, lymph nodes will be noted and included in the flap with preservation of the small vessels supplying 

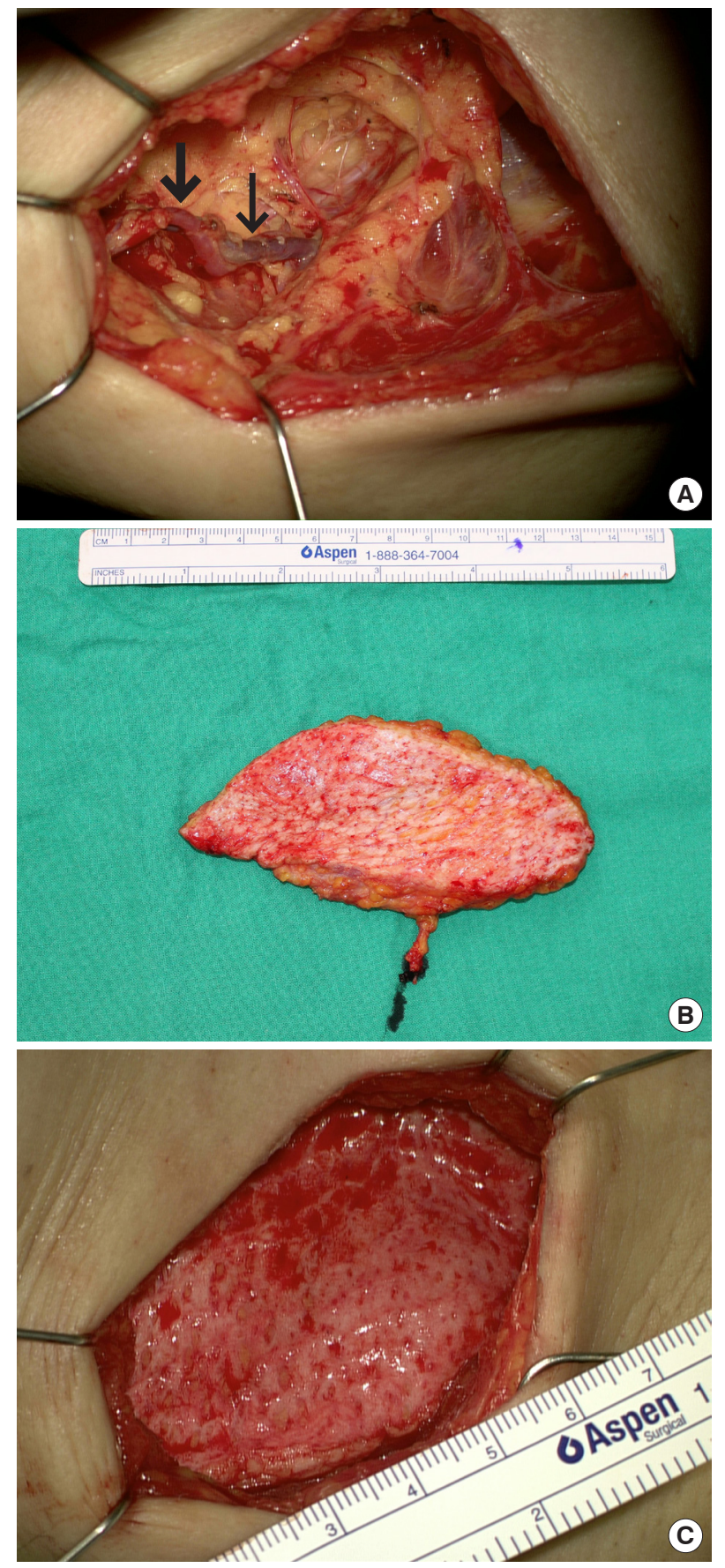

Fig. 3. Axillary scar removal and lymph node transfer. (A) After axillary scar tissue removal, a fresh fat tissue margin was achieved. The thoracodorsal artery (thick arrow) and vein (thin arrow) were dissected as recipient vessels. (B) A $15 \times 5 \mathrm{~cm}$ superficial circumflex iliac artery flap was harvested. (C) Dead space in the axillary pocket was obliterated with the superficial circumflex iliac artery flap.

the lymph node (Fig. 3B).

It is important to obliterate the entire dead space, and during flap positioning, the contact between the flap and peripheral fat layer should be reconstructed (Fig. 3C). The flap is anchored to the chest wall and axilla. As hematoma can cause fibrosis of the

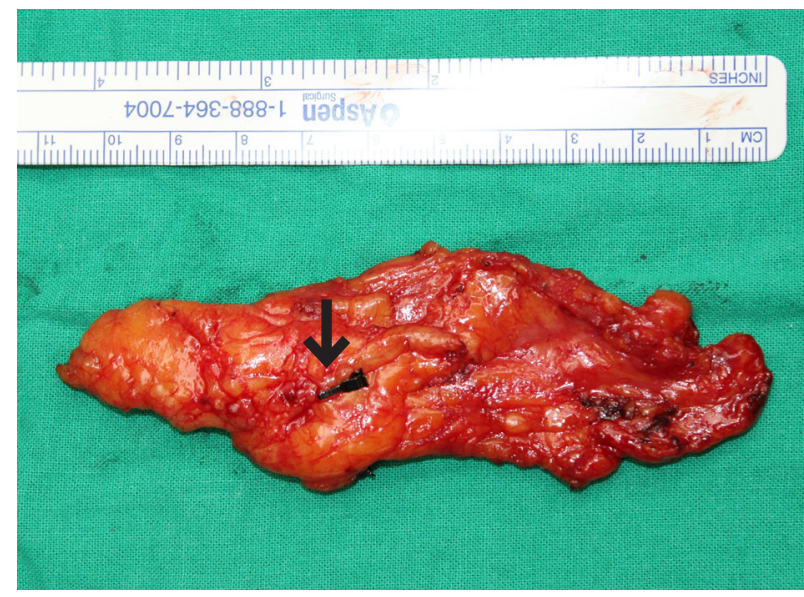

Fig. 4. For simultaneous breast reconstruction, the superficial circumflex iliac artery (arrow) flap includes a deep fat layer and lymph node. The skin and superficial fat layer are preserved at the donor site.

tissue again, meticulous hemostasis is required and insertion of a drain is recommended to prevent hematoma collection. Flap monitoring is performed using ultrasonography or a small monitoring flap. Ambulation begins on postoperative day 1 and the arm is maintained in the slightly abducted position using an arm sling to prevent compression at the pedicle site.

\section{Simultaneous breast reconstruction}

If a patient with lymphedema needs to undergo simultaneous breast reconstruction, the approach for lymphedema surgery can differ. For breast reconstruction, a deep inferior epigastric artery perforator (DIEP) free flap is selected. Conventionally, many centers attach the groin lymph node within the DIEP flap along the inferior margin of the DIEP flap as a single flap during the DIEP flap harvest [4]. However, in our experience, without separating the groin lymph node from the DIEP flap, it becomes difficult to inset the flap and to position the groin lymph node in the right space. Thus, the superficial circumflex iliac artery perforator flap is harvested separately from the DIEP flap. For a symmetric scar on the abdomen, the skin and superficial fat layer of the groin area are preserved at the donor site and only the deep fat layer and lymph node are harvested (Fig. 4). To minimize seroma collection at the donor site, negative-pressure wound therapy is applied to the groin donor site for 5 days [23]. In such cases, the serratus branch is usually used as the recipient vessel to preserve the main thoracodorsal artery and vein as a lifeboat in the case of DIEP flap compromise.

\section{DISCUSSION}

To provide an adequate surgical solution for lymphedema patients, the approach needs to be individualized. For a physiolog- 


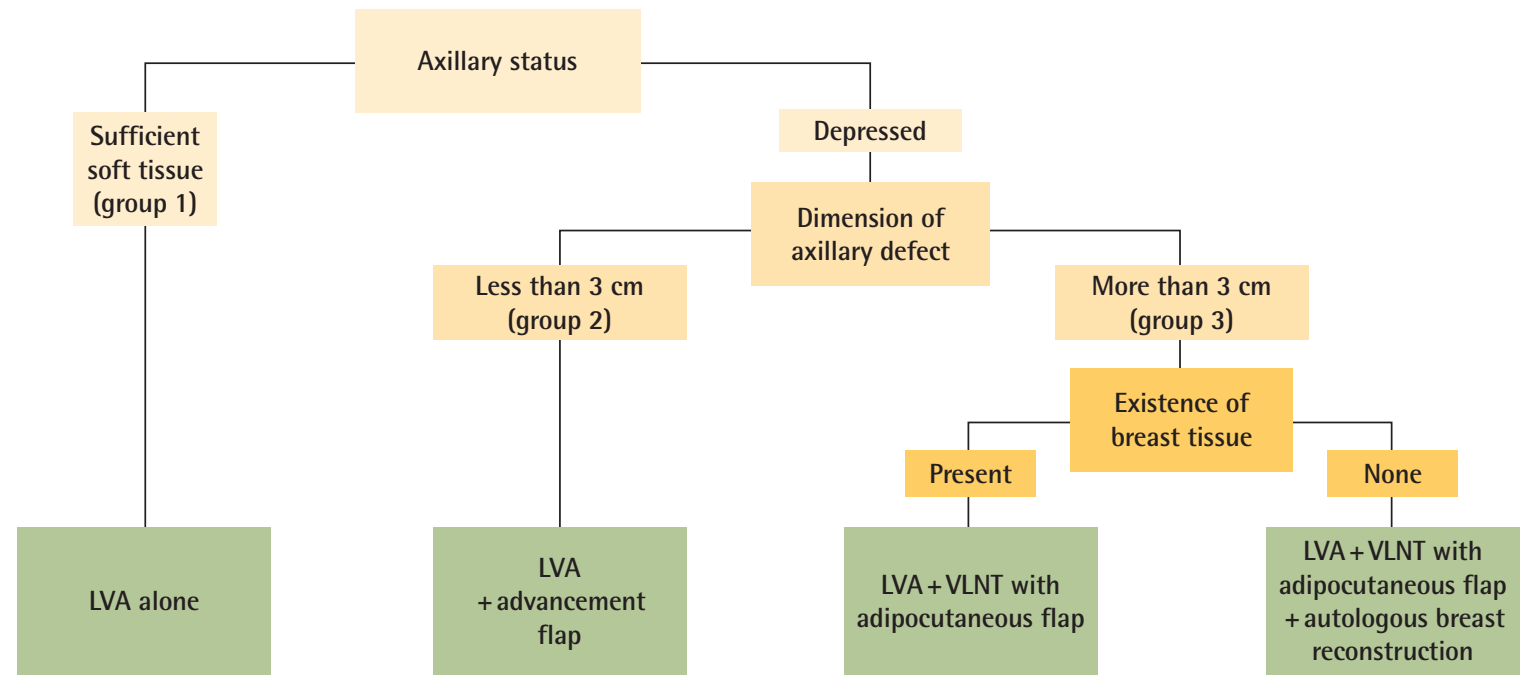

Fig. 5. Algorithm for surgical planning for breast cancer-related lymphedema patients. LVA, lymphaticovenous anastomosis; VLNT, vascularized lymph node transfer.
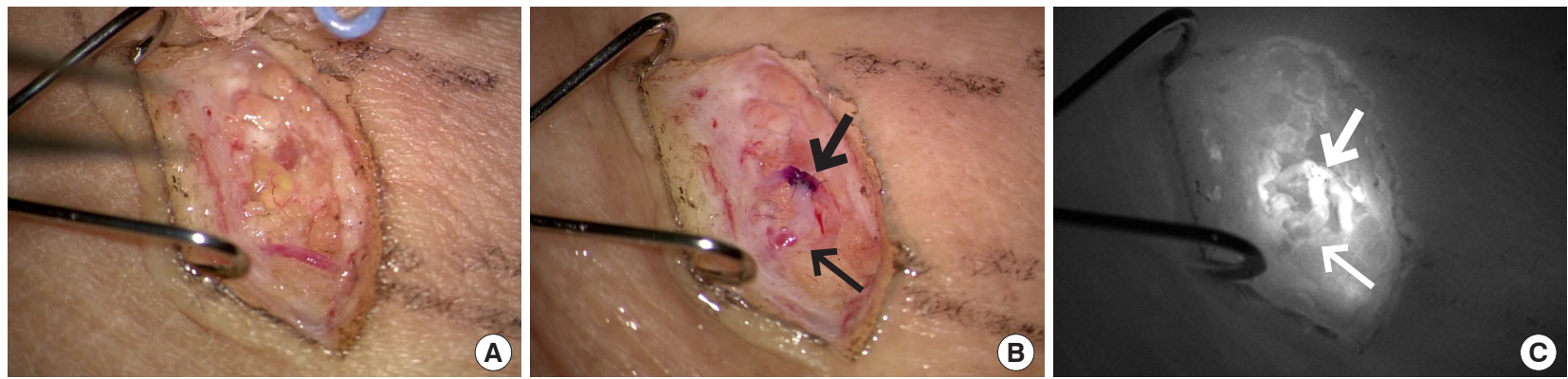

Fig. 6. Lymphaticovenous anastomosis in advanced lymphedema patient. (A) Even though there was severe fibrosis on the lobular septum and fat lobule itself, a 0.65- $\mathrm{mm}$ ectatic lymphatic was preserved from sclerosis. (B) Side-to-end lymphaticovenous anastomosis was performed to a $0.8 \mathrm{~mm}$ vein. (C) Indocyanine green drained into the anastomosed vein. Thick arrow is lymphatics and thin arrow is superficial vein.

ical surgical solution, one must think about the pathology of the obstructed site and provide a site-specific solution in addition to LVA. Fig. 5 provides an algorithm for the approach in lymphedema patients who will be considered for physiological surgical solutions.

In our experience, LVA can be performed in most patients regardless of the duration of lymphedema or the International Society of Lymphology (ISL) stage. Functioning lymphatics can be found in most BCRL patients. Patients with ISL stage 3, even if the fibrosis of the peripheral tissue is severe, frequently exhibit functioning lymphatics, although they may be fewer in number. Although the process of finding functioning lymphatics using ultrasonography is time-consuming, the reliability and predictability are very high, making surgery efficient. In patients with ISL stage 3 , by using functional lymphatics, the color of the vein changes to the lymphatic fluid color after LVA (Fig. 6).

In addition to LVA, the process for axillary scar removal is important. Unless there is very supple and unscarred axilla, most patients undergo some form of scar release. If the lymphatics are injured during the initial oncologic surgery, the lymphatic vessels contract, the defect is void of fat, and the resultant scars lead to a harsh environment for lymphatic regeneration. Furthermore, the scar tissue can compress the axillary veins, increasing the venous pressure and making the outcomes after LVA less favorable. Therefore, in order to treat lymphedema properly, these scars, which form barriers against lymphatic regeneration, must be well removed.

After scar tissue removal, the strategy for preventing the reformation of scar tissue must be followed by obliterating the dead space with fat and lymph nodes. Thus, a wider scar has a higher need for lymph node flaps. When simultaneous breast reconstruction is planned, one should not compromise the overall aesthetic outcome for breast reconstruction or positioning of the lymph node flap.

Even though liposuction is a treatment option for advanced lymphedema patients, liposuction is not recommended as a 
first-line treatment option as it can disrupt the remnant lymphatics. Studies have proven the effect of LVA in advanced-stage lymphedema. Therefore, physiological surgery should be tried as a first-line treatment option. At our center, liposuction is performed only for patients in whom physiological surgery had no effect. When liposuction was indicated, aggressive liposuction was performed under a tourniquet, as the entire subcutaneous fat layer should be removed. After liposuction, compressive bandaging should be performed before releasing the tourniquet.

There is still no exact algorithm for the surgical treatment of BCRL patients [24]. Because of the lack of knowledge about the exact pathophysiology of the disease, it is unsurprising that the algorithm shown here is primarily based on personal experiences. This paper introduces an algorithm based on addressing the pathology, with individualization depending on the severity of BCRL. Algorithms will always evolve and change, and this algorithm is one of many that surgeons can use.

\section{CONCLUSION}

By deciding the surgical plan for BCRL based on each patient's pathophysiology, optimal outcomes can be achieved. Depending on each patient's pathophysiology, LVA, scar removal, vascularized lymph node transfer with a sufficient adipocutaneous flap, and breast reconstruction should be planned.

\section{NOTES}

\section{Conflict of interest}

Hyunsuk Peter Suh, Changsik John Pak, and Joon Pio Hong are editorial board members of the journal but were not involved in the peer reviewer selection, evaluation, or decision process of this article. No other potential conflicts of interest relevant to this article were reported.

\section{Author contribution}

Conceptualization: JG Kwon, HP Suh, JP Hong. Data curation: JG Kwon, DW Hong. Writing - original draft: JG Kwon, JP Hong. Writing - review \& editing: HP Suh, CJ Pak, JP Hong.

\section{ORCID}

Jin Geun Kwon https://orcid.org/0000-0002-5873-3077

Hyunsuk Peter Suh https://orcid.org/0000-0003-1706-5516

Joon Pio Hong https://orcid.org/0000-0002-6208-9704

\section{REFERENCES}

1. DiSipio T, Rye S, Newman B, et al. Incidence of unilateral arm lymphoedema after breast cancer: a systematic review and meta-analysis. Lancet Oncol 2013;14:500-15.

2. Gillespie TC, Sayegh HE, Brunelle CL, et al. Breast cancerrelated lymphedema: risk factors, precautionary measures, and treatments. Gland Surg 2018;7:379-403.

3. Masia J, Pons G, Nardulli ML. Combined surgical treatment in breast cancer-related lymphedema. J Reconstr Microsurg 2016;32:16-27.

4. Schaverien MV, Coroneos CJ. Surgical treatment of lymphedema. Plast Reconstr Surg 2019;144:738-58.

5. Chang DW, Masia J, Garza R 3rd, et al. Lymphedema: surgical and medical therapy. Plast Reconstr Surg 2016;138(3 Suppl):209S-218S.

6. Cha HG, Oh TM, Cho MJ, et al. Changing the paradigm: lymphovenous anastomosis in advanced stage lower extremity lymphedema. Plast Reconstr Surg 2021;147:199207.

7. Cho MJ, Kwon JG, Pak CJ, et al. The role of duplex ultrasound in microsurgical reconstruction: review and technical considerations. J Reconstr Microsurg 2020;36:514-21.

8. Forte AJ, Boczar D, Kassis S, et al. Use of magnetic resonance imaging for evaluation of therapeutic response in breast cancer-related lymphedema: a systematic review. Arch Plast Surg 2020;47:305-9.

9. Suami H, Scaglioni MF. Anatomy of the lymphatic system and the lymphosome concept with reference to lymphedema. Semin Plast Surg 2018;32:5-11.

10. Hara H, Mihara M, Seki Y, et al. Comparison of indocyanine green lymphographic findings with the conditions of collecting lymphatic vessels of limbs in patients with lymphedema. Plast Reconstr Surg 2013;132:1612-8.

11. Aljindan FK, Lin CY, Cheng MH. Comparison of outcomes between side-to-end and end-to-end lymphovenous anastomoses for early-grade extremity lymphedema. Plast Reconstr Surg 2019; 144:486-96.

12. Pereira N, Lee YH, Suh Y, et al. Cumulative experience in lymphovenous anastomosis for lymphedema treatment: the learning curve effect on the overall outcome. J Reconstr Microsurg 2018;34:735-41.

13. Iyer D, Jannaway M, Yang Y, et al. Lymphatic valves and lymph flow in cancer-related lymphedema. Cancers (Basel) 2020;12:2297.

14. Olszewski WL. Contractility patterns of human leg lymphatics in various stages of obstructive lymphedema. Ann N Y Acad Sci 2008;1131:110-8.

15. Seki Y, Kajikawa A, Yamamoto T, et al. The dynamic-lymphaticovenular anastomosis method for breast cancer treatment-related lymphedema: creation of functional lymphati- 
covenular anastomoses with use of preoperative dynamic ultrasonography. J Plast Reconstr Aesthet Surg 2019;72:6270.

16. Kataru RP, Wiser I, Baik JE, et al. Fibrosis and secondary lymphedema: chicken or egg? Transl Res 2019;209:68-76.

17. Ly CL, Kataru RP, Mehrara BJ. Inflammatory manifestations of lymphedema. Int J Mol Sci 2017;18:171.

18. Sapountzis S, Nicoli F, Chilgar R, et al. Evidence-based analysis of lymph node transfer in postmastectomy upper extremity lymphedema. Arch Plast Surg 2013;40:450-1.

19. Suh HSP, Jeong HH, Choi DH, et al. Study of the medial superficial perforator of the superficial circumflex iliac artery perforator flap using computed tomographic angiography and surgical anatomy in 142 patients. Plast Reconstr Surg 2017;139:738-48.

20. Dayan JH, Dayan E, Smith ML. Reverse lymphatic mapping: a new technique for maximizing safety in vascularized lymph node transfer. Plast Reconstr Surg 2015;135:277-85.
21. Goh TLH, Park SW, Cho JY, et al. The search for the ideal thin skin flap: superficial circumflex iliac artery perforator flap: a review of 210 cases. Plast Reconstr Surg 2015;135: 592-601.

22. Hong JP, Sun SH, Ben-Nakhi M. Modified superficial circumflex iliac artery perforator flap and supermicrosurgery technique for lower extremity reconstruction: a new approach for moderate-sized defects. Ann Plast Surg 2013;71: 380-3.

23. Peter Suh HS, Hong JP. Effects of incisional negative-pressure wound therapy on primary closed defects after superficial circumflex iliac artery perforator flap harvest: randomized controlled study. Plast Reconstr Surg 2016;138:133340.

24. Lee KT, Lim SY, Pyun JK, et al. Improvement of upper extremity lymphedema after delayed breast reconstruction with an extended latissimus dorsi myocutaneous flap. Arch Plast Surg 2012;39:154-7. 